Supplemental Figure S1: Discrimination between Lys trimethylation and acetylation in ambiguous candidates.

For each site with a trimethyl-Lys to be examined, we considered the PSM of highest score and the corresponding acquisition run.

Summary of the examined ambiguities:

\begin{tabular}{|c|c|c|c|c|c|}
\hline Protein name & TAIR accession & Site & $\begin{array}{c}\text { Precursor Error } \\
(\mathrm{ppm})\end{array}$ & $\begin{array}{c}\text { Neutral loss } \\
(-59)\end{array}$ & Decision \\
\hline FBA1 & AT2G21330.1 & K395 & 1.385 & yes & accept Me3 \\
\hline eEF-1A & AT1G07920.1 & K187 & 5.121 & yes & accept Me3 \\
\hline GAPA2 & AT1G12900.1 & K314 & -7.962 & no & reject Me3 \\
\hline RBCL & AtCg00490 & K32 & -6.131 & yes & ambiguous \\
\hline RBCL & AtCg00490 & K236 & -5.208 & yes & ambiguous \\
\hline Tic62 & AT3G18890.1 & K79 & -7.947 & yes & ambiguous \\
\hline SDH & AT5G39410.1 & K6 & -1.729 & yes & ambiguous \\
\hline PRPL11 & AT1G32990.1 & K109 & 7.941 & yes & accept Me3 \\
\hline- & AT2G33090.1 & K79 & 5.803 & yes & accept Me3 \\
\hline
\end{tabular}

Error distribution graphs: distribution of the mass measurement errors within the acquisition run for all PSMs having a score $>40$. Abscissa: error values in ppm; coordinates: number of PSMs.

Blue line: true error for the candidate trimethyl-PSM to be examined.

Red line: artificial error for the same PSM if one substitutes trimethylation for acetylation.

MS/MS spectra: fragmentation spectra and ions tables of trimethyl-PSM candidates extracted from Mascot interpretation. 


\section{FBA1, AT2G21330.1}

YTGEGESEEAKEGMFVK ${ }_{395}$ GYTY, expmz 2448.071248

Interpreted with: M14, Dioxidation (M); K17, Trimethyl (K)
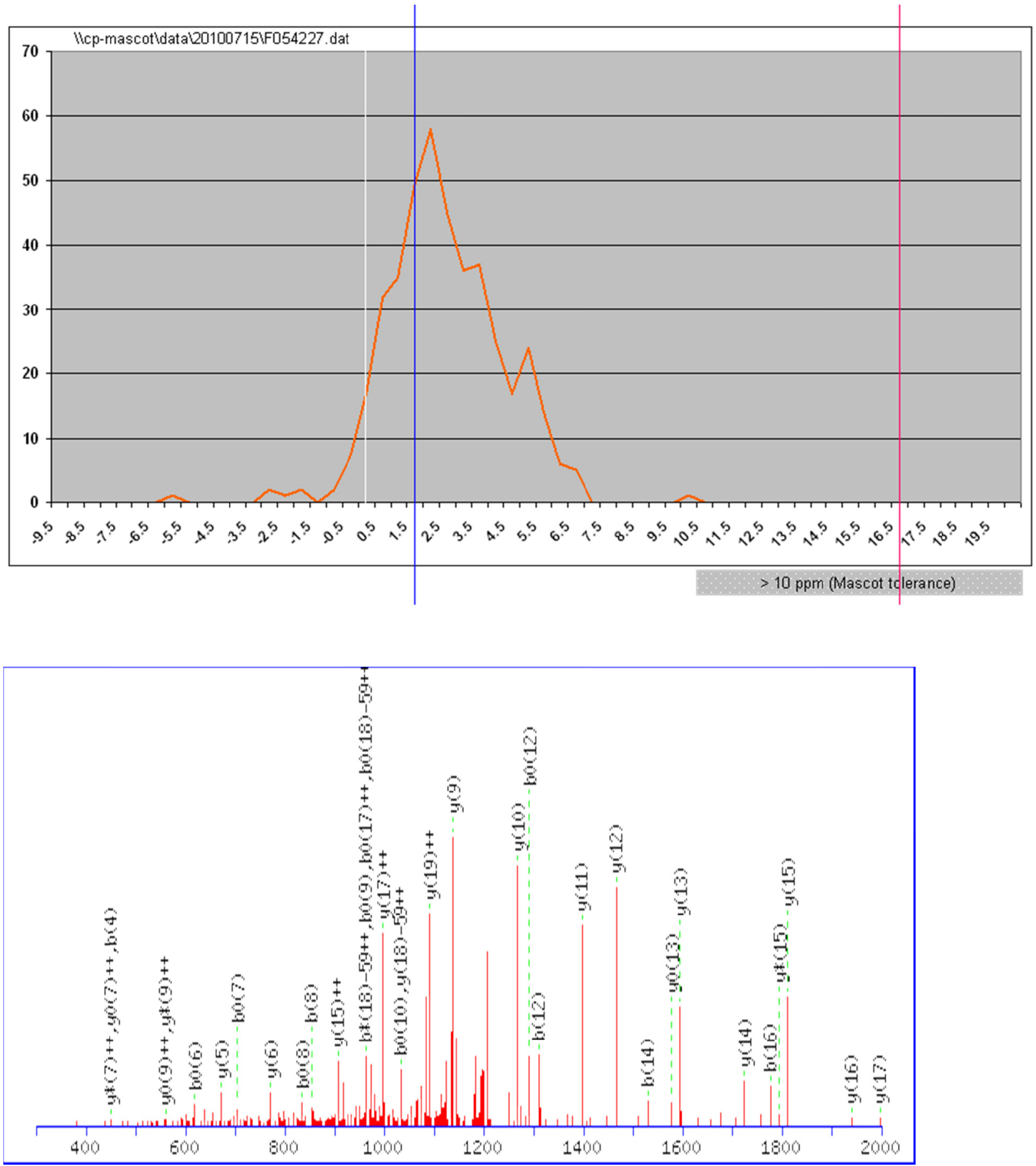


\begin{tabular}{|c|c|c|c|c|c|c|c|c|c|c|c|c|c|c|}
\hline \# & b & $\mathbf{b}^{++}$ & b* & $b^{*++}$ & $\mathbf{b}^{0}$ & $\mathbf{b}^{0++}$ & Seq. & $\mathbf{y}$ & $\mathbf{y}^{++}$ & $\mathbf{y}^{*}$ & $\mathbf{y}^{*++}$ & $\mathbf{y}^{0}$ & $\mathbf{y}^{0++}$ & \# \\
\hline 1 & 164.0706 & 82.5389 & & & & & $\mathbf{Y}$ & & & & & & & 21 \\
\hline 2 & 265.1183 & 133.0628 & & & 247.1077 & 124.0575 & $\mathbf{T}$ & 2286.0118 & 1143.5096 & 2268.9853 & 1134.9963 & 2268.0013 & 1134.5043 & 20 \\
\hline 3 & 322.1397 & 161.5735 & & & 304.1292 & 152.5682 & G & 2184.9642 & 1092.9857 & 2167.9376 & 1084.4724 & 2166.9536 & 1083.9804 & 19 \\
\hline 4 & 451.1823 & 226.0948 & & & 433.1718 & 217.0895 & $\mathbf{E}$ & 2127.9427 & 1064.4750 & 2110.9161 & 1055.9617 & 2109.9321 & 1055.4697 & 18 \\
\hline 5 & 508.2038 & 254.6055 & & & 490.1932 & 245.6003 & G & 1998.9001 & 999.9537 & 1981.8736 & 991.4404 & 1980.8895 & 990.9484 & 17 \\
\hline 7 & 724.2784 & 362.6429 & & & 706.2679 & 353.6376 & $\mathbf{S}$ & 1812.8360 & 906.9217 & 1795.8095 & 898.4084 & 1794.8255 & 897.9164 & 15 \\
\hline 8 & 853.3210 & 427.1641 & & & 835.3105 & 418.1589 & $\mathbf{E}$ & 1725.8040 & 863.4056 & 1708.7775 & 854.8924 & 1707.7935 & 854.4004 & 14 \\
\hline 9 & 982.3636 & 491.6854 & & & 964.3530 & 482.6802 & $\mathbf{E}$ & 1596.7614 & 798.8844 & 1579.7349 & 790.3711 & 1578.7509 & 789.8791 & 13 \\
\hline 10 & 1053.4007 & 527.2040 & & & 1035.3902 & 518.1987 & $\mathbf{A}$ & 1467.7188 & 734.3631 & 1450.6923 & 725.8498 & 1449.7083 & 725.3578 & 12 \\
\hline 11 & 1181.4957 & 591.2515 & 1164.4691 & 582.7382 & 1163.4851 & 582.2462 & $\mathbf{K}$ & 1396.6817 & 698.8445 & 1379.6552 & 690.3312 & 1378.6712 & 689.8392 & 11 \\
\hline 13 & 1367.5597 & 684.2835 & 1350.5332 & 675.7702 & 1349.5492 & 675.2782 & G & 1139.5442 & 570.2757 & 1122.5176 & 561.7624 & 1121.5336 & 561.2704 & 9 \\
\hline 14 & 1530.5901 & 765.7987 & 1513.5635 & 757.2854 & 1512.5795 & 756.7934 & M & 1082.5227 & 541.7650 & 1065.4961 & 533.2517 & 1064.5121 & 532.7597 & 8 \\
\hline 15 & 1677.6585 & 839.3329 & 1660.6319 & 830.8196 & 1659.6479 & 830.3276 & $\mathbf{F}$ & 919.4924 & 460.2498 & 902.4658 & 451.7366 & 901.4818 & 451.2445 & 7 \\
\hline 16 & 1776.7269 & 888.8671 & 1759.7003 & 880.3538 & 1758.7163 & 879.8618 & $\mathbf{V}$ & 772.4240 & 386.7156 & 755.3974 & 378.2023 & 754.4134 & 377.7103 & 6 \\
\hline 17 & 1946.8688 & 973.9380 & 1929.8423 & 965.4248 & 1928.8582 & 964.9328 & $\mathbf{K}$ & 673.3556 & 337.1814 & 656.3290 & 328.6681 & 655.3450 & 328.1761 & 5 \\
\hline 18 & 2003.8903 & 1002.4488 & 1986.8637 & 993.9355 & 1985.8797 & 993.4435 & G & 503.2136 & 252.1105 & & & 485.2031 & 243.1052 & 4 \\
\hline 19 & 2166.9536 & 1083.9804 & 2149.9270 & 1075.4672 & $|2148.9430|$ & 1074.9752 & $\mathbf{Y}$ & 446.1922 & 223.5997 & & & 428.1816 & 214.5944 & 3 \\
\hline 20 & 2268.0013 & 1134.5043 & 2250.9747 & 1125.9910 & 2249.9907 & 1125.4990 & $\mathbf{T}$ & 283.1288 & 142.0681 & & & 265.1183 & 133.0628 & 2 \\
\hline 21 & & & & & & & $\mathbf{Y}$ & 182.0812 & 91.5442 & & & & & 1 \\
\hline
\end{tabular}


eEF-1A, AT1G07920.1

VGYNPDK $_{187}$ IPFVPISGFEGDNMIER, expmz 2751.371892

Interpreted with: K7, Trimethyl (K); M21, Oxidation (M)
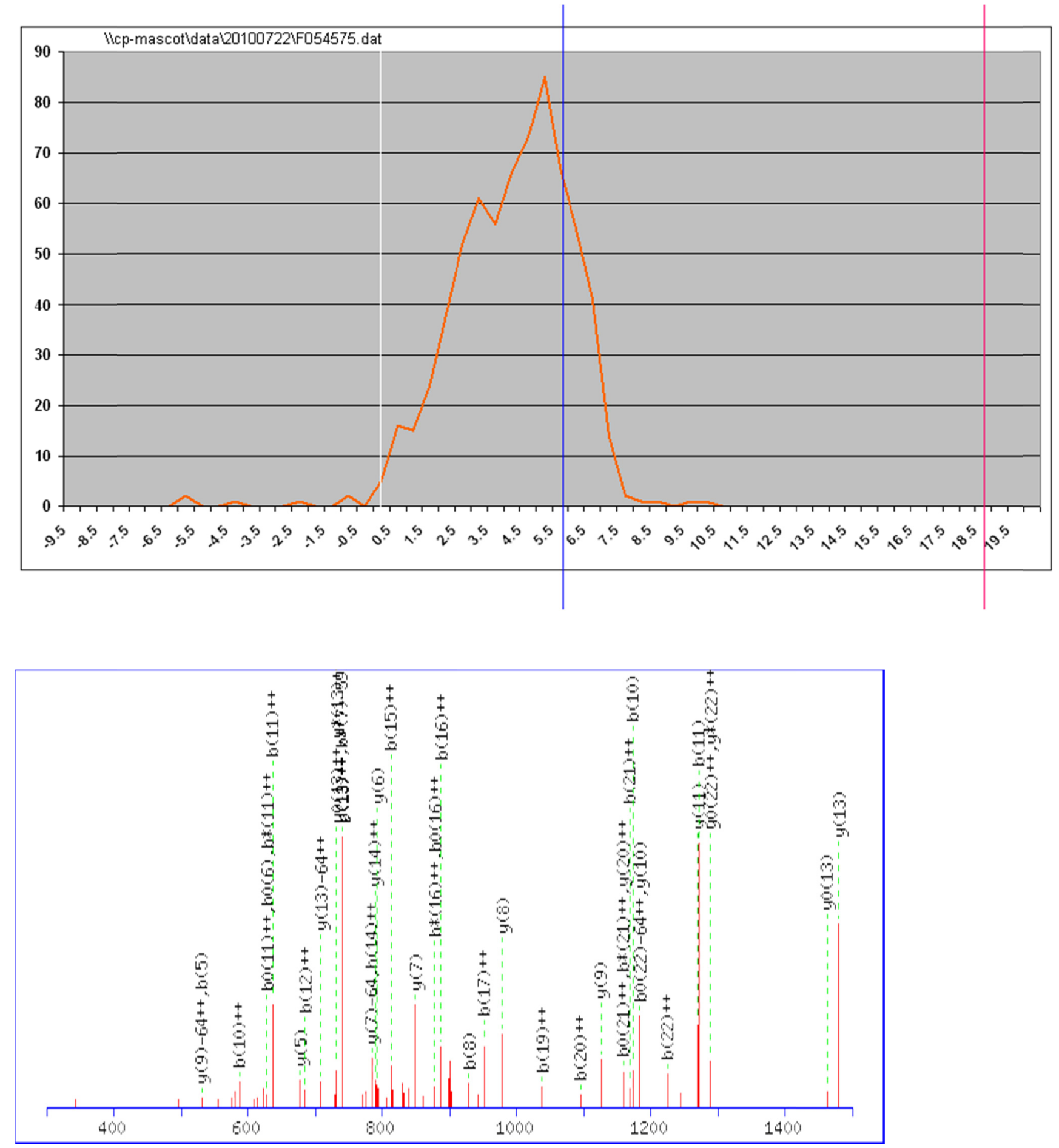
Zoom in the $720-760$ ppm range:

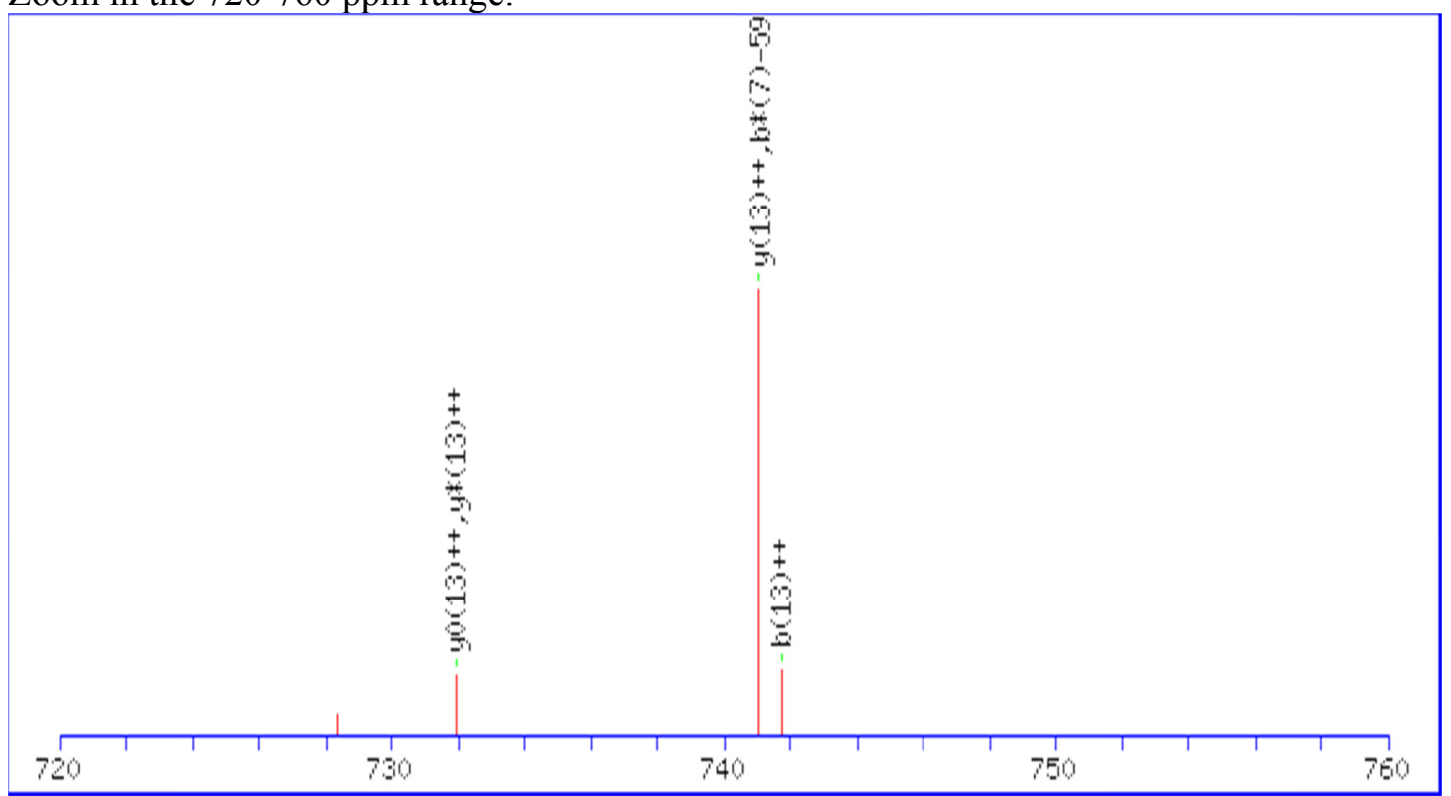

\begin{tabular}{|c|c|c|c|c|c|c|c|c|c|c|c|c|c|c|}
\hline$\#$ & b & $\mathbf{b}^{++}$ & & & $\mathbf{b}^{0}$ & & Seq. & $\mathbf{y}$ & & & & $\mathbf{y}^{0}$ & $\mathbf{y}^{0++}$ & \# \\
\hline 1 & 100.0757 & 50.5415 & & & & & $\mathbf{V}$ & & & & & & & 24 \\
\hline 2 & 157.0972 & 79.0522 & & & & & G & & & 2636.2701 & & 2635.2861 & & 23 \\
\hline 3 & 320.1605 & 160.5839 & & & & & $\mathbf{Y}$ & 2596.2752 & 1298.6412 & 2579.2487 & 1290.1280 & 2578.2646 & 1289.6360 & 22 \\
\hline 4 & 434.2034 & 217.6053 & 417.1769 & & & & $\mathbf{N}$ & & 1217.1096 & 2416.1853 & $12: 08.5963$ & 2415.2013 & 1208.1043 & 21 \\
\hline 5 & 531.2562 & 266.1317 & & & & & $\mathbf{P}$ & 2319.1689 & 1160.0881 & 2302.1424 & 1151.5748 & 2301.1584 & 1151.0828 & 20 \\
\hline 7 & 816.4250 & 408.7162 & 799.3985 & & 798.4145 & & $\mathbf{K}$ & 2107.0892 & 1054.0483 & 2090.0627 & 10145.5350 & 2089.0787 & 1045.0430 & 18 \\
\hline 8 & 929.5091 & 465.2582 & & & & & I & & & 1919.9208 & & 1918.9368 & 959.9720 & 17 \\
\hline 9 & 1026.5619 & 513.7846 & 1009.5353 & & 1008.5513 & & $\mathbf{P}$ & & & & & & 903.4300 & 16 \\
\hline 10 & 1173.6303 & 587.3188 & & & & & $\mathbf{F}$ & & & & & & & 15 \\
\hline 11 & 1272.6987 & 636.8530 & & & & & $\mathbf{V}$ & & & & & & & 14 \\
\hline 13 & & 741.9214 & & & & & I & & & & & & & 12 \\
\hline 14 & & 785.4374 & & & & & $\mathbf{S}$ & & & & & & & 11 \\
\hline 15 & 1626.8890 & 813.9481 & & & & & G & & & & & & & 10 \\
\hline 16 & 1773.9574 & 887.4823 & & & & & $\mathbf{F}$ & & & & & & & 9 \\
\hline 17 & 1903.0000 & 952.0036 & 1885.9735 & 943.4904 & 1884.9894 & & $\mathbf{E}$ & & & & & & & 8 \\
\hline 18 & 1960.0215 & 980.5144 & 1942.9949 & 972.0011 & 1942.0109 & 971.5091 & G & 850.3723 & & 833.3458 & & & & 7 \\
\hline 19 & 2075.0484 & 1038.0278 & 2058.0219 & 1029.5146 & 2057.0379 & 1029.0226 & D & 793.3509 & 397.1791 & 776.3243 & 3.88 .6658 & 775.3403 & & 6 \\
\hline 20 & 2189.0913 & 1095.0493 & 2172.0648 & 1086.5360 & 2171.0808 & 1086.0440 & $\mathbf{N}$ & 678.3239 & 339.6656 & 661.2974 & 3.31 .1523 & 660.3134 & 330.6603 & 5 \\
\hline 21 & 2336.1267 & 1168.5670 & 2319.1002 & 1160.0537 & 2318.1162 & 1159.5617 & M & 564.2810 & 282.6441 & 547.2545 & 274.1309 & 546.2704 & 273.6389 & 4 \\
\hline 22 & 2449.2108 & 1225.1090 & 2432.1843 & 1216.5958 & 2431.2002 & 1216.1038 & I & 417.2456 & 209.1264 & 400.2191 & $2: 00.6132$ & 399.2350 & 200.1212 & 3 \\
\hline 23 & 2578.2534 & 1289.6303 & 2561.2269 & 1281.1171 & 2560.2428 & 1280.6251 & $\mathbf{E}$ & 304.1615 & 152.5844 & 287.1350 & 144.0711 & 286.1510 & 143.5791 & 2 \\
\hline
\end{tabular}




\section{GAPA2, AT1G12900.1}

VPTPNVSVVDLVVQVSK ${ }_{314}$, expmz 1821.046688

Interpreted with: K17, Trimethyl (K)
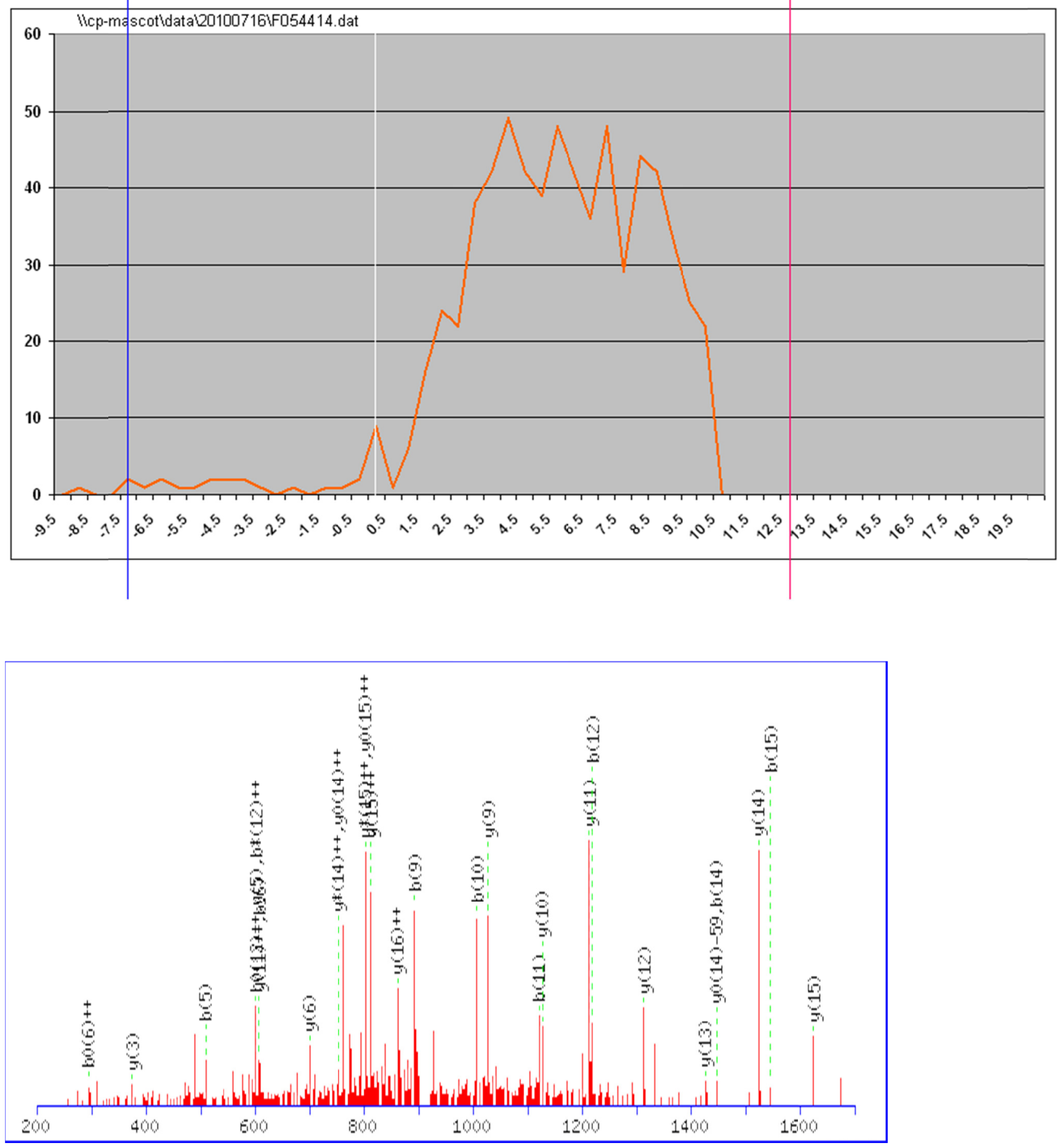


\begin{tabular}{|c|c|c|c|c|c|c|c|c|c|c|c|c|c|c|}
\hline \# & b & $\mathbf{b}^{++}$ & $\mathbf{b}^{*}$ & $\mathbf{b}^{*++}$ & $\mathbf{b}^{0}$ & $\mathbf{b}^{0++}$ & Seq. & $\mathbf{y}$ & $\mathbf{y}^{++}$ & $y^{*}$ & $\mathbf{y}^{*++}$ & $\mathbf{y}^{0}$ & $\mathbf{y}^{0++}$ & \# \\
\hline 1 & 100.0757 & 50.5415 & & & & & $\mathbf{V}$ & & & & & & & 17 \\
\hline 2 & 197.1285 & 99.0679 & & & & & $\mathbf{P}$ & 1723.0000 & 862.0036 & 1705.9735 & 853.4904 & 1704.9894 & 852.9984 & 16 \\
\hline 3 & 298.1761 & 149.5917 & & & 280.1656 & 140.5864 & $\mathbf{T}$ & $\mid 1625.9472$ & 813.4773 & 1608.9207 & 804.9640 & 1607.9367 & 804.4720 & 15 \\
\hline 4 & 395.2289 & 198.1181 & & & 377.2183 & 189.1128 & $\mathbf{P}$ & 1524.8996 & 762.9534 & 1507.8730 & 754.4401 & 1506.8890 & |753.9481 & 14 \\
\hline 5 & 509.2718 & 255.1396 & 492.2453 & 246.6263 & 491.2613 & 246.1343 & $\mathbf{N}$ & $\mid 1427.8468$ & $\mid 714.4270$ & 1410.8203 & 705.9138 & 1409.8362 & $\mid 705.4218$ & 13 \\
\hline 6 & 608.3402 & 304.6738 & 591.3137 & 296.1605 & 590.3297 & 295.6685 & V & $\mid 1313.8039$ & 657.4056 & 1296.7773 & 648.8923 & 1295.7933 & 648.4003 & 12 \\
\hline 7 & 695.3723 & 348.1898 & 678.3457 & 339.6765 & 677.3617 & 339.1845 & $\mathbf{S}$ & $\mid 1214.7355$ & 607.8714 & 1197.7089 & 599.3581 & 1196.7249 & $598.8661 \mid$ & 11 \\
\hline 8 & 794.4407 & 397.7240 & 777.4141 & 389.2107 & 776.4301 & 388.7187 & V & $|1127.7034|$ & 564.3554 & 1110.6769 & 555.8421 & 1109.6929 & $|555.3501|$ & 10 \\
\hline 9 & 893.5091 & 447.2582 & 876.4825 & 438.7449 & 875.4985 & 438.2529 & V & $\mid 1028.6350$ & 514.8211 & 1011.6085 & 506.3079 & 1010.6245 & 505.8159 & 9 \\
\hline 10 & 1008.5360 & 504.7717 & 991.5095 & 496.2584 & 990.5255 & 495.7664 & D & 929.5666 & 465.2869 & 912.5401 & 456.7737 & 911.5560 & $|456.2817|$ & 8 \\
\hline 11 & 1121.6201 & 561.3137 & $\mid 1104.5936$ & 552.8004 & 1103.6095 & 552.3084 & $\mathbf{L}$ & 814.5397 & 407.7735 & 797.5131 & 399.2602 & 796.5291 & 398.7682 & 7 \\
\hline 12 & 1220.6885 & 610.8479 & $|1203.6620|$ & 602.3346 & $\mid 1202.6780$ & 601.8426 & V & 701.4556 & 351.2314 & 684.4291 & 342.7182 & 683.4450 & 342.2262 & 6 \\
\hline 13 & 1319.7569 & 660.3821 & 1302.7304 & 651.8688 & 1301.7464 & 651.3768 & V & 602.3872 & 301.6972 & 585.3606 & 293.1840 & 584.3766 & 292.6919 & 5 \\
\hline 14 & 1447.8155 & 724.4114 & $\mid 1430.7890$ & 715.8981 & $\mid 1429.8049$ & 715.4061 & Q & 503.3188 & 252.1630 & 486.2922 & 243.6498 & 485.3082 & 243.1577 & 4 \\
\hline 15 & 1546.8839 & 773.9456 & $\mid 1529.8574$ & 765.4323 & 1528.8734 & 764.9403 & V & 375.2602 & 188.1337 & 358.2336 & 179.6205 & 357.2496 & 179.1285 & 3 \\
\hline 16 & 1633.9159 & 817.4616 & 1616.8894 & 808.9483 & 1615.9054 & 808.4563 & $\mathbf{S}$ & 276.1918 & 138.5995 & 259.1652 & 130.0863 & 258.1812 & 129.5942 & 2 \\
\hline 17 & & & & & & & K & 189.1598 & 95.0835 & 172.1332 & 86.5702 & & & 1 \\
\hline
\end{tabular}




\section{RBCL, AtCg00490; K32}

LTYYTPEYETK ${ }_{32}$ DTDILAAFR, expmz 2451.205902

Interpreted with: K11, Trimethyl (K)
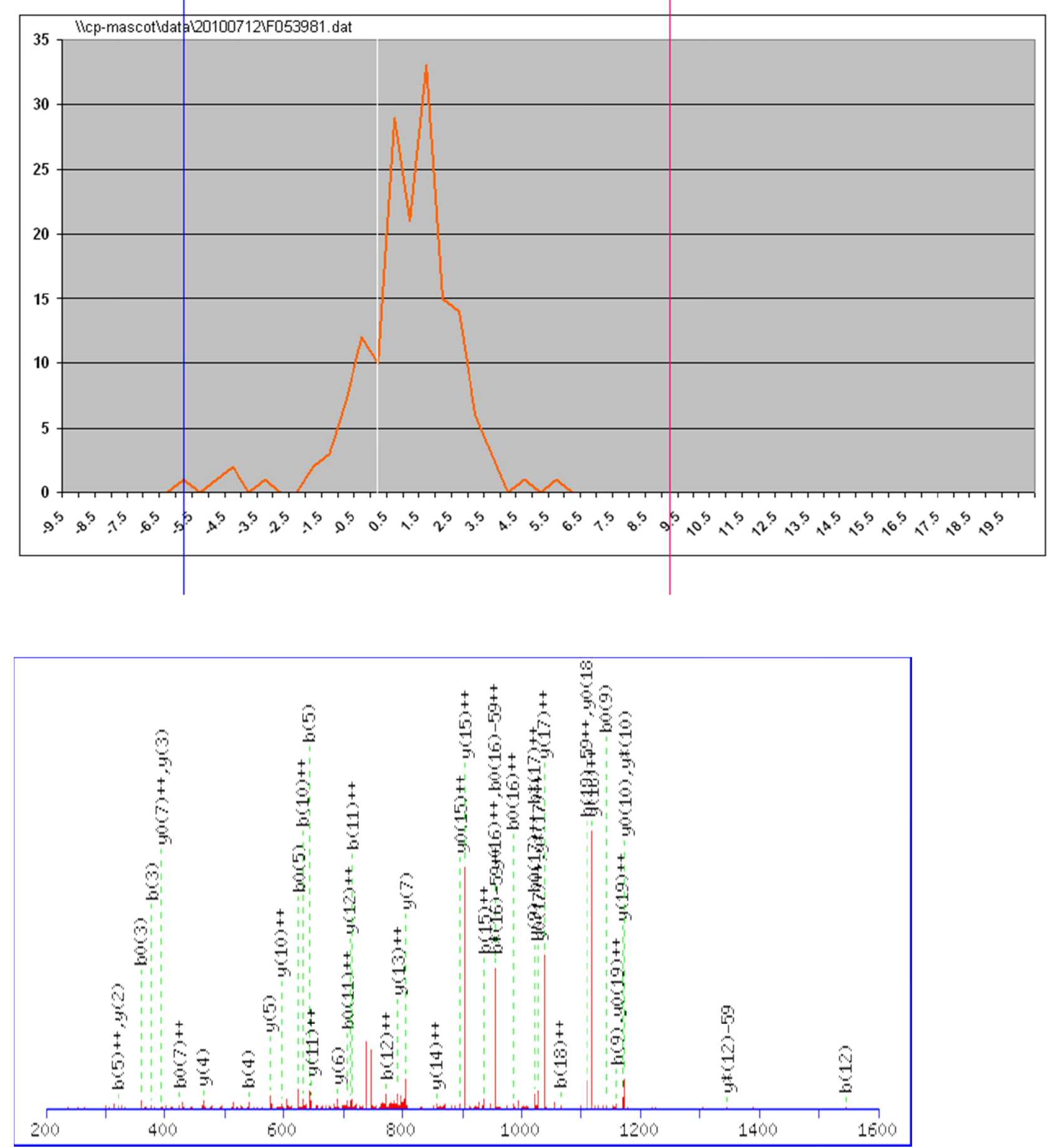
Zoom in the $800-1200 \mathrm{ppm}$ range:

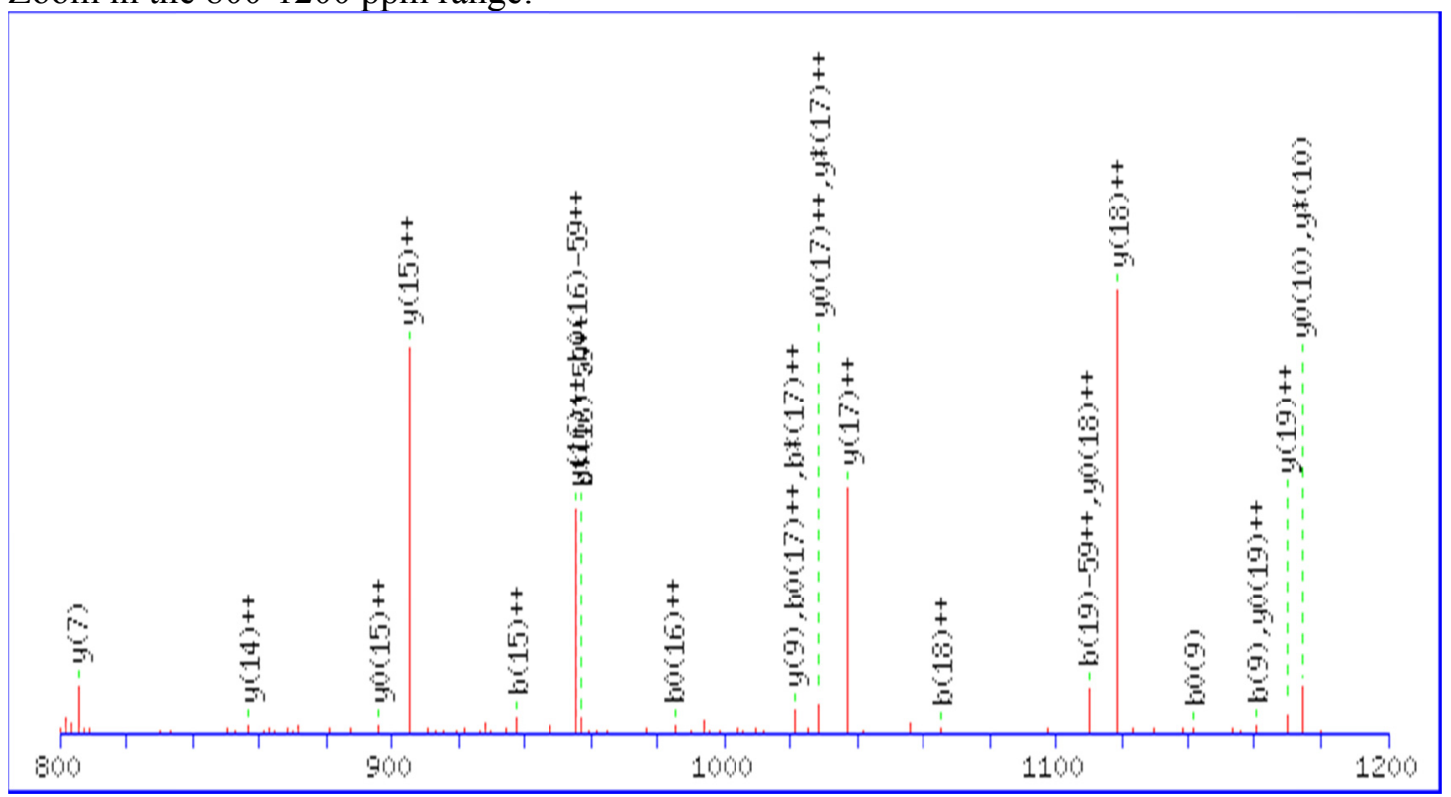

\begin{tabular}{|c|c|c|c|c|c|c|c|c|c|c|c|c|c|c|}
\hline \# & b & $\mathbf{b}^{++}$ & $\mathbf{b}^{*}$ & $\mathbf{b}^{*++}$ & $\mathbf{b}^{0}$ & $\mathbf{b}^{0++}$ & Seq. & $\mathbf{y}$ & $\mathbf{y}^{++}$ & $\mathbf{y}^{*}$ & $\mathbf{y}^{*++}$ & $\mathbf{y}^{0}$ & $\mathbf{y}^{0++}$ & \# \\
\hline 1 & 114.0913 & 57.5493 & & & & & $\mathbf{L}$ & & & & & & & 20 \\
\hline 2 & 215.1390 & 108.0731 & & & 197.1285 & 99.0679 & $T$ & 2339.1442 & 1170.0757 & 2322.1176 & 1161.5625 & 2321.1336 & 1161.0704 & 19 \\
\hline 3 & 378.2023 & 189.6048 & & & 360.1918 & 180.5995 & $\mathbf{Y}$ & 2238.0965 & 1119.5519 & 2221.0699 & 1111.0386 & 2220.0859 & 1110.5466 & 18 \\
\hline 4 & 541.2657 & 271.1365 & & & 523.2551 & 262.1312 & $\mathbf{Y}$ & 2075.0332 & 1038.0202 & 2058.0066 & 10129.5069 & 2057.0226 & 1029.0149 & 17 \\
\hline 5 & 642.3134 & 321.6603 & & & 624.3028 & 312.6550 & $\mathbf{T}$ & 1911.9698 & 956.4886 & 1894.9433 & 9147.9753 & 1893.9593 & 947.4833 & 16 \\
\hline 7 & 868.4087 & 434.7080 & & & 850.3981 & 425.7027 & $\mathbf{E}$ & 1713.8694 & 857.4383 & 1696.8428 & $8: 48.9251$ & 1695.8588 & 848.4331 & 14 \\
\hline 8 & 1031.4720 & 516.2397 & & & 1013.4615 & 507.2344 & $\mathbf{Y}$ & 1584.8268 & 792.9170 & 1567.8003 & 784.4038 & 1566.8162 & 783.9118 & 13 \\
\hline 9 & 1160.5146 & 580.7610 & & & 1142.5041 & 571.7557 & $\mathbf{E}$ & 1421.7635 & 711.3854 & 1404.7369 & 702.8721 & 1403.7529 & 702.3801 & 12 \\
\hline 10 & 1261.5623 & 631.2848 & & & 1243.5517 & 622.2795 & $\mathbf{T}$ & 1292.7209 & 646.8641 & 1275.6943 & 6.38 .3508 & 1274.7103 & 637.8588 & 11 \\
\hline 11 & 1431.7042 & 716.3558 & 1414.6777 & 707.8425 & 1413.6937 & 707.3505 & $\mathbf{K}$ & 1191.6732 & 596.3402 & 1174.6467 & $5: 87.8270$ & 1173.6626 & 587.3350 & 10 \\
\hline 13 & 1647.7788 & 824.3931 & 1630.7523 & 815.8798 & 1629.7683 & 815.3878 & $\mathbf{T}$ & 906.5043 & 453.7558 & 889.4778 & 445.2425 & 888.4938 & 444.7505 & 8 \\
\hline 14 & 1762.8058 & 881.9065 & 1745.7792 & 873.3933 & 1744.7952 & 872.9013 & D & 805.4567 & 403.2320 & 788.4301 & $3,94.7187$ & 787.4461 & 394.2267 & 7 \\
\hline 15 & 1875.8899 & 938.4486 & 1858.8633 & 929.9353 & 1857.8793 & 929.4433 & I & 690.4297 & 345.7185 & 673.4032 & 3.37 .2052 & & & 6 \\
\hline 16 & 1988.9739 & 994.9906 & 1971.9474 & 986.4773 & 1970.9634 & 985.9853 & $\mathbf{L}$ & 577.3457 & 289.1765 & 560.3191 & 280.6632 & & & 5 \\
\hline 17 & 2060.0110 & 1030.5092 & 2042.9845 & 1021.9959 & 2042.0005 & 1021.5039 & $\mathbf{A}$ & 464.2616 & 232.6344 & 447.2350 & 224.1212 & & & 4 \\
\hline 18 & 2131.0481 & 1066.0277 & 2114.0216 & 1057.5144 & 2113.0376 & 1057.0224 & $\mathbf{A}$ & 393.2245 & 197.1159 & 376.1979 & 188.6026 & & & 3 \\
\hline 19 & 2278.1166 & 1139.5619 & 2261.0900 & 1131.0486 & 2260.1060 & 1130.5566 & $\mathbf{F}$ & 322.1874 & 161.5973 & 305.1608 & 153.0840 & & & 2 \\
\hline 20 & & & & & & & $\mathbf{R}$ & 175.1190 & 88.0631 & 158.0924 & 79.5498 & & & 1 \\
\hline
\end{tabular}




\section{RBCL, AtCg00490; K236}

SQAETGEIK ${ }_{236}$ GHYLNATAGTCEEMIK, expmz 2802.254142

Interpreted with: K9, Trimethyl (K); M23, Dioxidation (M)
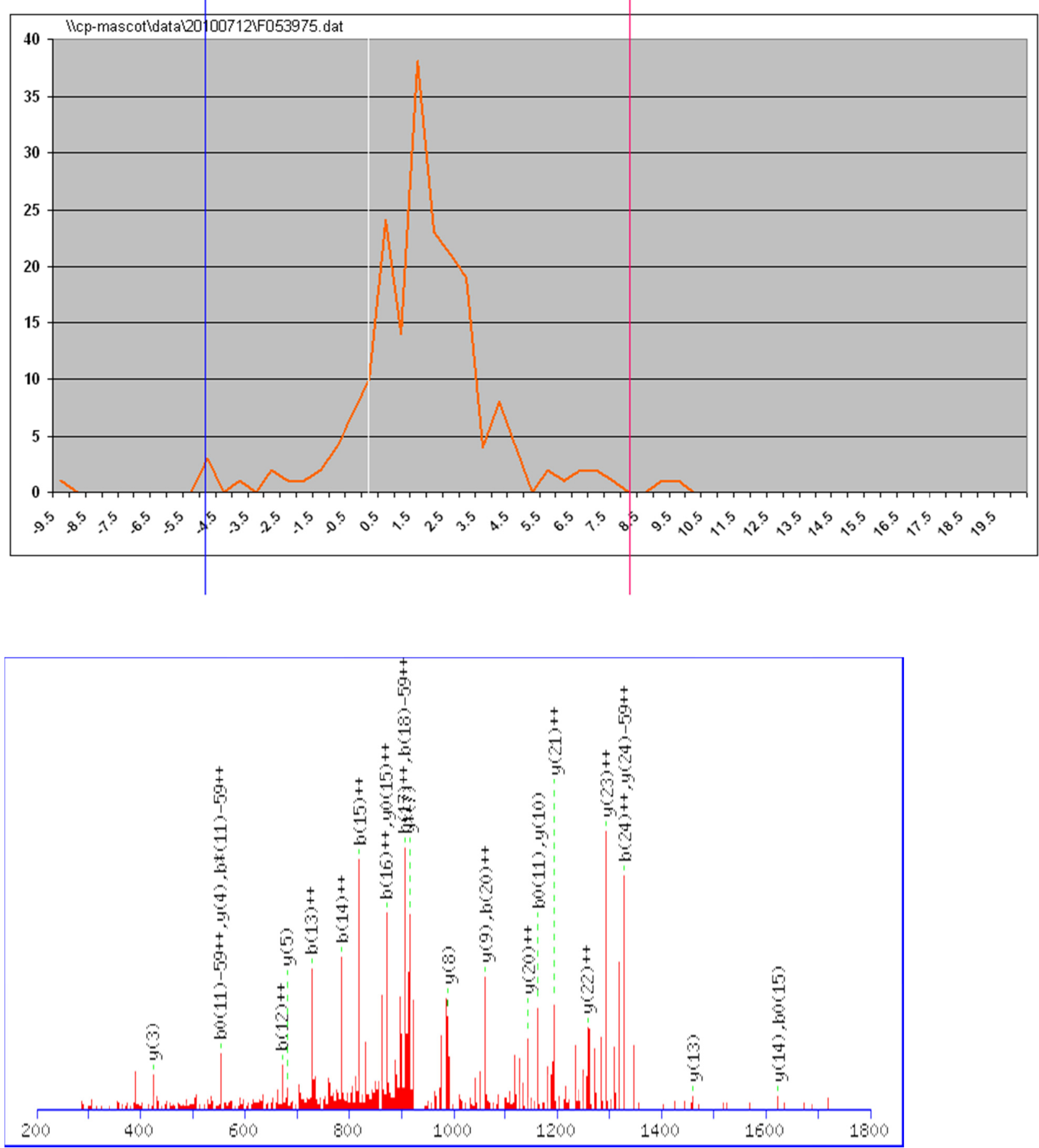
Zoom in the 500-1400 ppm range:

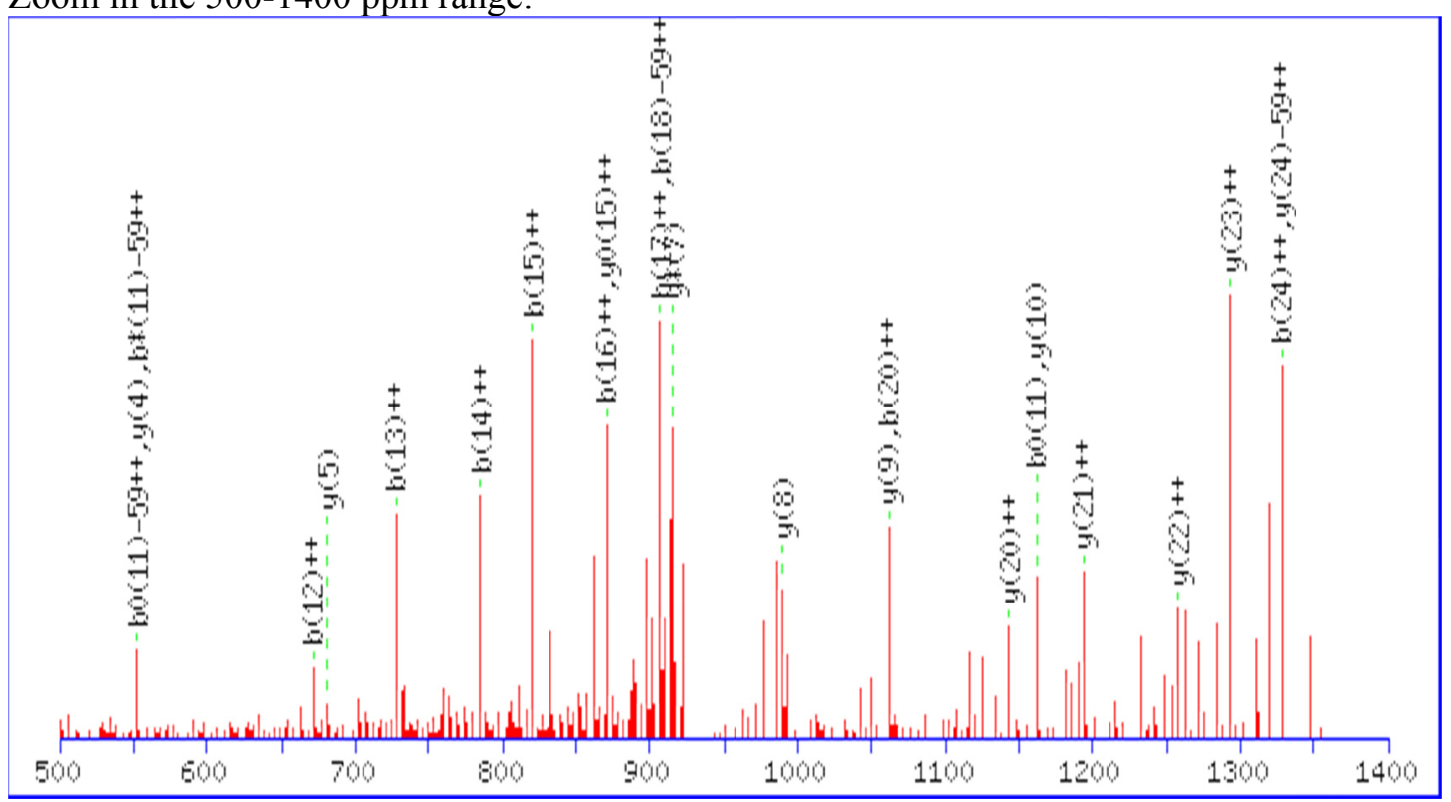

\begin{tabular}{|c|c|c|c|c|c|c|c|c|c|c|c|c|c|c|}
\hline \# & b & $\mathbf{b}^{++}$ & b* & $\mathbf{b}^{*++}$ & $\mathbf{b}^{0}$ & $\mathbf{b}^{0++}$ & Seq. & $\mathbf{y}$ & $\mathbf{y}^{++}$ & $\mathbf{y}^{*}$ & $y^{*++}$ & $\mathbf{y}^{0}$ & $\mathbf{y}^{0++}$ & \# \\
\hline 1 & 88.0393 & 44.5233 & & & 70.0287 & 35.5180 & $\mathbf{S}$ & & & & & & & 25 \\
\hline 2 & 216.0979 & 108.5526 & 199.0713 & 100.0393 & 198.0873 & 99.5473 & $\mathbf{Q}$ & 2716.2440 & 1358.6257 & 2699.2175 & 1350.1124 & 2698.2335 & 1349.6204 & 24 \\
\hline 3 & 287.1350 & 144.0711 & 270.1084 & 135.5579 & 269.1244 & 135.0659 & $\mathbf{A}$ & 2588.1855 & 1294.5964 & 2571.1589 & $12: 86.0831$ & 2570.1749 & 1285.5911 & 23 \\
\hline 4 & 416.1776 & 208.5924 & 399.1510 & 200.0792 & 398.1670 & 199.5872 & $\mathbf{E}$ & 2517.1483 & 1259.0778 & 2500.1218 & 12.50 .5645 & 2499.1378 & 1250.0725 & 22 \\
\hline 5 & 517.2253 & 259.1163 & 500.1987 & 250.6030 & 499.2147 & 250.1110 & $\mathbf{T}$ & 2388.1058 & 1194.5565 & 2371.0792 & 1186.0432 & 2370.0952 & 1185.5512 & 21 \\
\hline 7 & 703.2893 & 352.1483 & 686.2628 & 343.6350 & 685.2788 & 343.1430 & $\mathbf{E}$ & 2230.0366 & 1115.5219 & 2213.0101 & 1107.0087 & 2212.0260 & 1106.5167 & 19 \\
\hline 8 & 816.3734 & 408.6903 & 799.3468 & 400.1771 & 798.3628 & 399.6851 & I & 2100.9940 & 1051.0006 & 2083.9675 & 10142.4874 & 2082.9835 & 1041.9954 & 18 \\
\hline 9 & 986.5153 & 493.7613 & 969.4888 & 485.2480 & 968.5047 & 484.7560 & $\mathbf{K}$ & 1987.9100 & 994.4586 & 1970.8834 & 9185.9453 & 1969.8994 & 985.4533 & 17 \\
\hline 10 & 1043.5368 & 522.2720 & 1026.5102 & 513.7587 & 1025.5262 & 513.2667 & G & 1817.7680 & 909.3877 & 1800.7415 & 900.8744 & 1799.7575 & 900.3824 & 16 \\
\hline 11 & 1180.5957 & 590.8015 & 1163.5691 & 582.2882 & 1162.5851 & 581.7962 & $\mathbf{H}$ & 1760.7466 & 880.8769 & 1743.7200 & $8: 72.3637$ & 1742.7360 & 871.8716 & 15 \\
\hline 13 & 1456.7431 & 728.8752 & 1439.7165 & 720.3619 & 1438.7325 & 719.8699 & $\mathbf{L}$ & 1460.6243 & 730.8158 & 1443.5978 & 722.3025 & 1442.6138 & 721.8105 & 13 \\
\hline 14 & 1570.7860 & 785.8966 & 1553.7594 & 777.3834 & 1552.7754 & 776.8914 & $\mathbf{N}$ & 1347.5403 & 674.2738 & 1330.5137 & $6,65.7605$ & 1329.5297 & 665.2685 & 12 \\
\hline 15 & 1641.8231 & 821.4152 & 1624.7966 & 812.9019 & 1623.8125 & 812.4099 & $\mathbf{A}$ & 1233.4973 & 617.2523 & 1216.4708 & 608.7390 & 1215.4868 & 608.2470 & 11 \\
\hline 16 & 1742.8708 & 871.9390 & 1725.8442 & 863.4258 & 1724.8602 & 862.9338 & $\mathbf{T}$ & 1162.4602 & 581.7338 & 1145.4337 & $5: 73.2205$ & 1144.4497 & 572.7285 & 10 \\
\hline 17 & 1813.9079 & 907.4576 & 1796.8814 & 898.9443 & 1795.8973 & 898.4523 & $\mathbf{A}$ & 1061.4126 & 531.2099 & 1044.3860 & $5: 22.6966$ & 1043.4020 & 522.2046 & 9 \\
\hline 18 & 1870.9294 & 935.9683 & 1853.9028 & 927.4550 & 1852.9188 & 926.9630 & G & 990.3754 & 495.6914 & 973.3489 & 487.1781 & 972.3649 & 486.6861 & 8 \\
\hline 19 & 1971.9770 & 986.4922 & 1954.9505 & 977.9789 & 1953.9665 & 977.4869 & $\mathbf{T}$ & 933.3540 & 467.1806 & 916.3274 & 458.6674 & 915.3434 & 458.1753 & 7 \\
\hline 20 & 2122.9710 & 1061.9891 & 2105.9444 & 1053.4759 & 2104.9604 & 1052.9838 & $\mathrm{C}$ & 832.3063 & 416.6568 & 815.2797 & 408.1435 & 814.2957 & 407.6515 & 6 \\
\hline 21 & 2252.0136 & 1126.5104 & 2234.9870 & 1117.9971 & 2234.0030 & 1117.5051 & $\mathbf{E}$ & 681.3124 & 341.1598 & 664.2858 & 332.6465 & 663.3018 & 332.1545 & 5 \\
\hline 22 & 2381.0562 & 1191.0317 & 2364.0296 & 1182.5184 & 2363.0456 & 1182.0264 & $\mathbf{E}$ & 552.2698 & 276.6385 & 535.2432 & 268.1253 & 534.2592 & 267.6332 & 4 \\
\hline 23 & 2544.0865 & 1272.5469 & 2527.0599 & 1264.0336 & 2526.0759 & 1263.5416 & M & 423.2272 & 212.1172 & 406.2006 & $2: 03.6040$ & & & 3 \\
\hline
\end{tabular}




\section{TIC62, AT3G18890.1}

ASSVVTEASPTNLNSK ${ }_{79}$ EEDLVFVAGATGK, mzexp 2962.489962

Interpreted with: K16, Trimethyl (K)
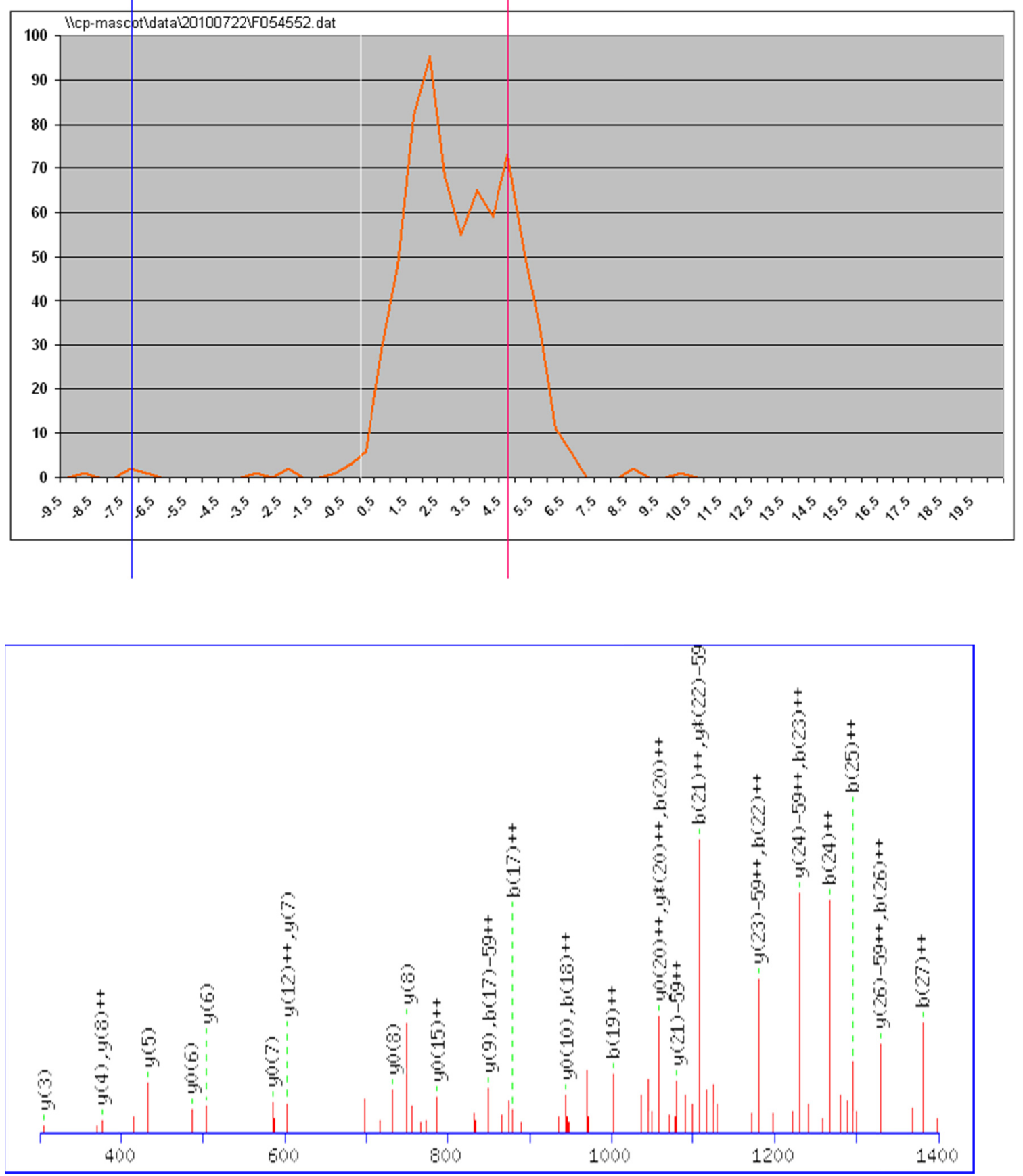


\begin{tabular}{|c|c|c|c|c|c|c|c|c|c|c|c|c|c|c|}
\hline$\#$ & b & $\mathbf{b}^{++}$ & $\mathbf{b}^{*}$ & $\mathbf{b}^{*++}$ & $\mathbf{b}^{0}$ & $\mathbf{b}^{0++}$ & Seq. & $\mathbf{y}$ & $\mathbf{y}^{++}$ & $y^{*}$ & $y^{*++}$ & $\mathbf{y}^{0}$ & $\mathbf{y}^{0++}$ & $\#$ \\
\hline 1 & 72.0444 & 36.5258 & & & & & $\mathbf{A}$ & & & & & & & 29 \\
\hline 2 & 159.0764 & 80.0418 & & & 141.0659 & 71.0366 & $\mathbf{S}$ & 2892.4837 & 1446.7455 & 2875.4571 & 1438.2322 & 2874.4731 & 1437.7402 & 28 \\
\hline 3 & 246.1084 & 123.5579 & & & 228.0979 & 114.5526 & $\mathbf{S}$ & 2805.4516 & 1403.2295 & 2788.4251 & 1394.7162 & 2787.4411 & 1394.2242 & 27 \\
\hline 4 & 345.1769 & 173.0921 & & & 327.1663 & 164.0868 & $\mathbf{V}$ & 2718.4196 & 1359.7134 & 2701.3931 & 1351.2002 & 2700.4091 & 1350.7082 & 26 \\
\hline 5 & 444.2453 & 222.6263 & & & 426.2347 & 213.6210 & $\mathbf{V}$ & 2619.3512 & 1310.1792 & 2602.3247 & 1301.6660 & 2601.3406 & 1301.1740 & 25 \\
\hline 7 & 674.3355 & 337.6714 & & & 656.3250 & 328.6661 & $\mathbf{E}$ & 2419.2351 & 1210.1212 & 2402.2086 & 1201.6079 & 2401.2245 & 1201.1159 & 23 \\
\hline 8 & 745.3727 & 373.1900 & & & 727.3621 & 364.1847 & $\mathbf{A}$ & 2290.1925 & 1145.5999 & 2273.1660 & 1137.0866 & 2272.1820 & 1136.5946 & 22 \\
\hline 9 & 832.4047 & 416.7060 & & & 814.3941 & 407.7007 & $\mathbf{S}$ & 2219.1554 & 1110.0813 & 2202.1289 & 1101.5681 & 2201.1448 & 1101.0761 & 21 \\
\hline 10 & 929.4575 & 465.2324 & & & 911.4469 & 456.2271 & $\mathbf{P}$ & 2132.1234 & 1066.5653 & 2115.0968 & 1058.0521 & 2114.1128 & 1057.5600 & 20 \\
\hline 11 & 1030.5051 & 515.7562 & & & 1012.4946 & 506.7509 & $\mathbf{T}$ & 2035.0706 & 1018.0389 & 2018.0441 & 1009.5257 & 2017.0600 & 1009.0337 & 19 \\
\hline 13 & 1257.6321 & 629.3197 & 1240.6056 & 620.8064 & 1239.6216 & 620.3144 & $\mathbf{L}$ & 1819.9800 & 910.4936 & 1802.9535 & 901.9804 & 1801.9694 & 901.4884 & 17 \\
\hline 14 & 1371.6750 & 686.3412 & 1354.6485 & 677.8279 & 1353.6645 & 677.3359 & $\mathbf{N}$ & 1706.8959 & 853.9516 & 1689.8694 & 845.4383 & 1688.8854 & 844.9463 & 16 \\
\hline 15 & 1458.7071 & 729.8572 & 1441.6805 & 721.3439 & 1440.6965 & 720.8519 & $\mathbf{S}$ & 1592.8530 & 796.9301 & 1575.8265 & 788.4169 & 1574.8424 & 787.9249 & 15 \\
\hline 16 & 1628.8490 & 814.9281 & 1611.8224 & 806.4149 & 1610.8384 & 805.9229 & $\mathbf{K}$ & 1505.8210 & 753.4141 & 1488.7944 & 744.9009 & 1487.8104 & 744.4088 & 14 \\
\hline 17 & 1757.8916 & 879.4494 & 1740.8650 & 870.9362 & 1739.8810 & 870.4441 & $\mathbf{E}$ & 1335.6791 & 668.3432 & 1318.6525 & 659.8299 & 1317.6685 & 659.3379 & 13 \\
\hline 18 & 1886.9342 & 943.9707 & 1869.9076 & 935.4575 & 1868.9236 & 934.9654 & $\mathbf{E}$ & 1206.6365 & 603.8219 & 1189.6099 & 595.3086 & 1188.6259 & 594.8166 & 12 \\
\hline 19 & 2001.9611 & 1001.4842 & 1984.9346 & 992.9709 & 1983.9506 & 992.4789 & D & 1077.5939 & 539.3006 & 1060.5673 & 530.7873 & 1059.5833 & 530.2953 & 11 \\
\hline 20 & 2115.0452 & 1058.0262 & 2098.0186 & 1049.5130 & 2097.0346 & 1049.0209 & $\mathbf{L}$ & 962.5669 & 481.7871 & 945.5404 & 473.2738 & 944.5564 & 472.7818 & 10 \\
\hline 21 & 2214.1136 & 1107.5604 & 2197.0870 & 1099.0472 & 2196.1030 & 1098.5552 & $\mathbf{V}$ & 849.4829 & 425.2451 & 832.4563 & 416.7318 & 831.4723 & 416.2398 & 9 \\
\hline 22 & 2361.1820 & 1181.0946 & 2344.1555 & 1172.5814 & 2343.1714 & 1172.0894 & $\mathbf{F}$ & 750.4145 & 375.7109 & 733.3879 & 367.1976 & 732.4039 & 366.7056 & 8 \\
\hline 23 & 2460.2504 & 1230.6289 & 2443.2239 & 1222.1156 & 2442.2399 & 1221.6236 & V & 603.3461 & 302.1767 & 586.3195 & 293.6634 & 585.3355 & 293.1714 & 7 \\
\hline 26 & 2659.3461 & 1330.1767 & 2642.3196 & 1321.6634 & 2641.3356 & 1321.1714 & $\mathbf{A}$ & 376.2191 & 188.6132 & 359.1925 & 180.0999 & 358.2085 & 179.6079 & 4 \\
\hline 27 & 2760.3938 & 1380.7005 & 2743.3672 & 1372.1873 & 2742.3832 & 1371.6953 & $\mathbf{T}$ & 305.1819 & 153.0946 & 288.1554 & 144.5813 & 287.1714 & 144.0893 & 3 \\
\hline 28 & 2817.4153 & 1409.2113 & 2800.3887 & 1400.6980 & 2799.4047 & 1400.2060 & $\mathbf{G}$ & 204.1343 & 102.5708 & 187.1077 & 94.0575 & & & 2 \\
\hline 29 & & & & & & & $\mathbf{K}$ & 147.1128 & 74.0600 & 130.0863 & 65.5468 & & & 1 \\
\hline
\end{tabular}




\section{SDH, AT5G39410.1}

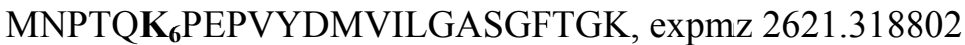

Interpreted with: K6, Trimethyl (K)
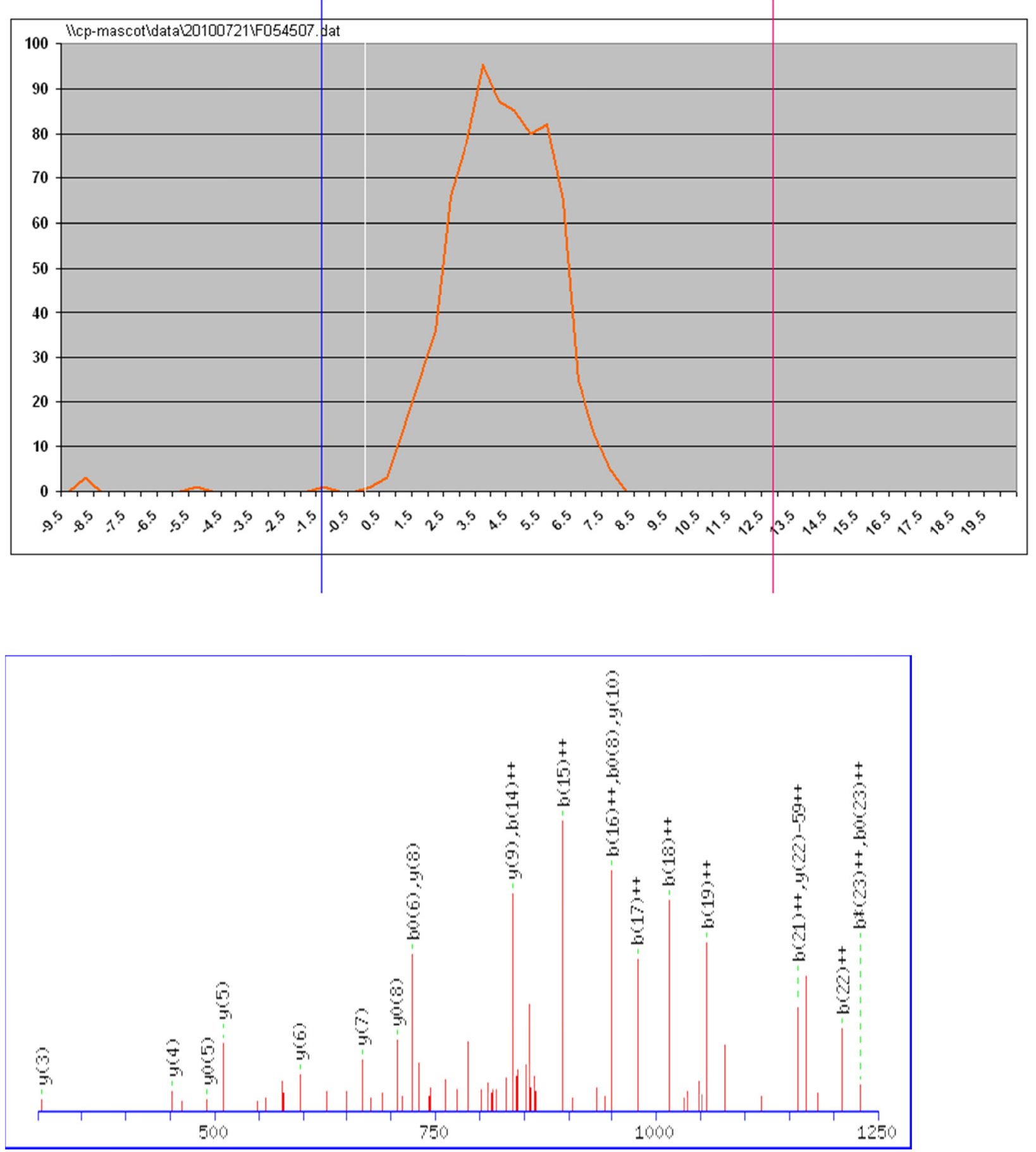


\begin{tabular}{|c|c|c|c|c|c|c|c|c|c|c|c|c|c|c|}
\hline \# & b & $\mathbf{b}^{++}$ & b* & & $\mathbf{b}^{0}$ & & eq. & $\mathbf{y}$ & & $\mathrm{y}^{*}$ & & $\mathbf{y}^{0}$ & & \# \\
\hline 1 & 132.0478 & 66.5275 & & & & & & & & & & & & 24 \\
\hline 2 & 246.0907 & 123.5490 & & & & & & & & 474.2636 & 37.6354 & 2473.2796 & 1237.1434 & 23 \\
\hline 3 & 343.1435 & & & 163.5621 & & & & 2377.2472 & 1189.1272 & 2360.2206 & 1180.6140 & 2359.2366 & 1180.1220 & 22 \\
\hline 4 & 444.1911 & 222.5992 & 427.1646 & 214.0859 & 426.1806 & & $\mathbf{T}$ & 2280.1944 & 1140.6009 & 2263.1679 & 1132.0876 & 2262.1839 & 1131.5956 & 21 \\
\hline 5 & 572.2497 & 286.6285 & 555.2232 & & & 277.6232 & & 2179.1468 & 1090.0770 & 2162.1202 & 1081.5637 & 2161.1362 & 1081.0717 & 20 \\
\hline 7 & 839.4444 & 420.2258 & & & & & & & 940.9768 & & 932.4635 & & 931.9715 & 18 \\
\hline 8 & 968.4870 & & & & & & & & & & & & & 17 \\
\hline 9 & 1065.5397 & 533.2735 & & & & & $\mathbf{P}$ & & & & & & & 16 \\
\hline 10 & 1164.6082 & 582.8077 & & & & & & & & & 770.8894 & & 770.3974 & 15 \\
\hline 11 & 1327.6715 & 664.3394 & & & & & & & & & & & 720.8632 & 14 \\
\hline 13 & 1573.7389 & 787.3731 & 1556.7124 & 778.8598 & 1555.7283 & & & & 590.8234 & 1163.6129 & 582.3101 & 1162.6289 & 581.8181 & 12 \\
\hline 14 & 1672.8073 & 836.9073 & 1655.7808 & 828.3940 & 1654.7968 & & $\mathbf{V}$ & & 525.3031 & 1032.5724 & 516.7898 & 1031.5884 & 516.2978 & 11 \\
\hline 15 & 1785.8914 & 893.4493 & 1768.8648 & 884.9361 & 1767.8808 & & I & & 475.7689 & 933.5040 & 467.2556 & 932.5200 & 466.7636 & 10 \\
\hline 16 & 1898.9755 & 949.9914 & 1881.9489 & 941.4781 & 1880.9649 & 940.9861 & $\mathbf{L}$ & 837.4465 & 419.2269 & 820.4199 & 410.7136 & 819.4359 & 410.2216 & 9 \\
\hline 17 & 1955.9969 & 978.5021 & 1938.9704 & 969.9888 & 1937.9864 & 969.4968 & G & 724.3624 & 362.6849 & 707.3359 & 354.1716 & 706.3519 & 353.6796 & 8 \\
\hline 18 & 2027.0340 & 1014.0207 & 2010.0075 & 1005.5074 & 2009.0235 & 1005.0154 & A & 667.3410 & 334.1741 & 650.3144 & 325.6608 & 649.3304 & 325.1688 & 7 \\
\hline 19 & 2114.0661 & 1057.5367 & 2097.0395 & 1049.0234 & 2096.0555 & 1048.5314 & $\mathbf{S}$ & 596.3039 & 298.6556 & 579.2773 & 290.1423 & 578.2933 & 289.6503 & 6 \\
\hline 20 & 2171.0875 & 1086.0474 & 2154.0610 & 1077.5341 & 2153.0770 & 1077.0421 & G & 509.2718 & 255.1396 & 492.2453 & 246.6263 & 491.2613 & 246.1343 & 5 \\
\hline 21 & 2318.1559 & 1159.5816 & 2301.1294 & 1151.0683 & 2300.1454 & 1150.5763 & $\mathbf{F}$ & 452.2504 & 226.6288 & 435.2238 & & & & 4 \\
\hline 22 & 2419.2036 & 1210.1054 & 2402.1771 & 1201.5922 & 2401.1931 & 1201.1002 & $\mathbf{T}$ & & 153.0946 & & & 287.1714 & 144.0893 & 3 \\
\hline 23 & 2476.2251 & 1238.6162 & 2459.1985 & 1230.1029 & 2458.2145 & 1229.6109 & G & 204.1343 & 102.5708 & & & & & 2 \\
\hline
\end{tabular}




\section{PRPL11, AT1G32990.1}

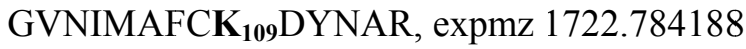

Interpreted with: M5, Dioxidation (M); K9, Trimethyl (K)
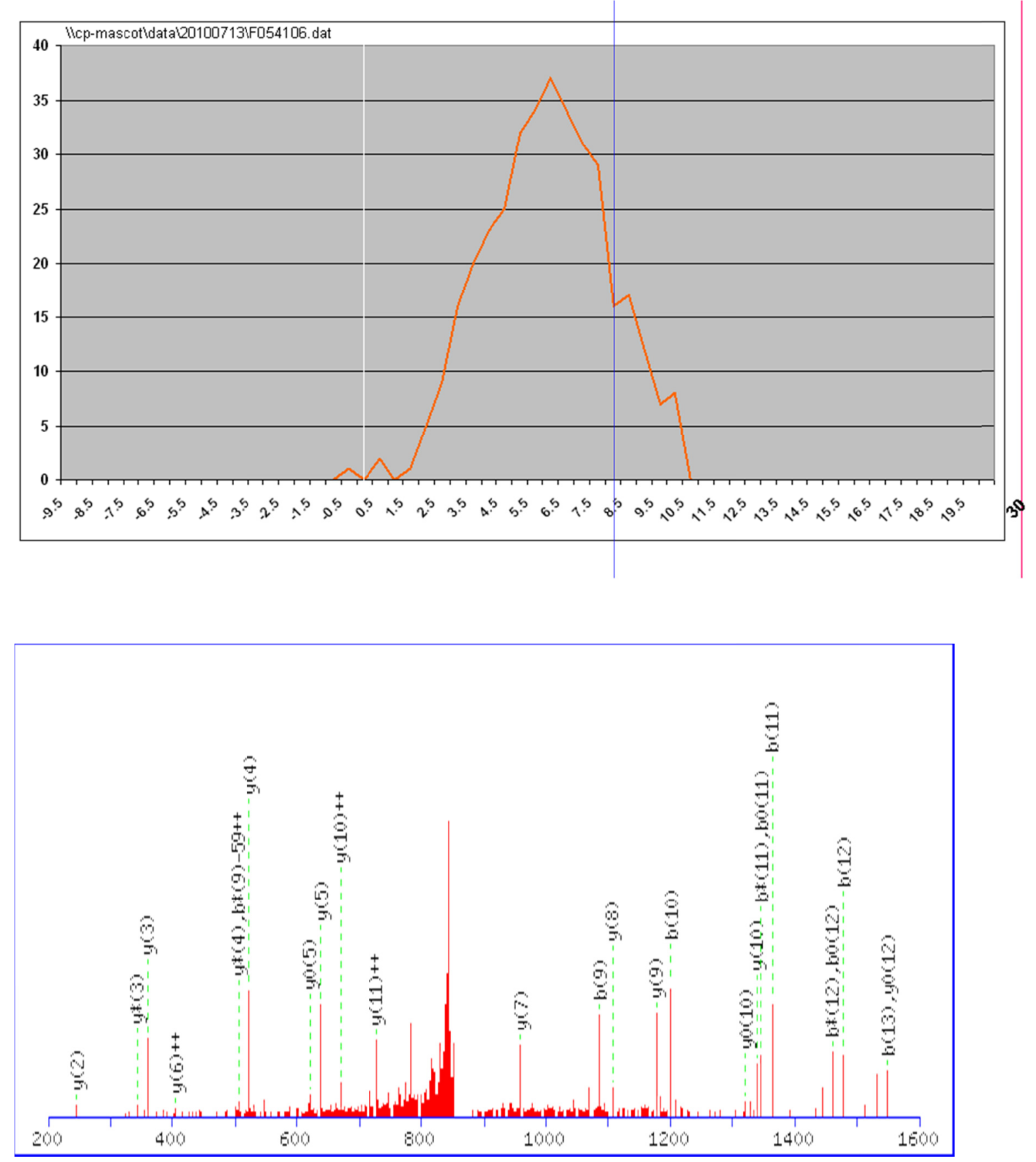


\begin{tabular}{|c|c|c|c|c|c|c|c|c|c|c|c|c|c|c|}
\hline$\#$ & b & $\mathbf{b}^{++}$ & $\mathbf{b}^{*}$ & $\mathbf{b}^{*++}$ & $\mathbf{b}^{0}$ & $\mathbf{b}^{0++}$ & Seq. & $\mathbf{y}$ & $\mathbf{y}^{++}$ & $\mathrm{y}^{*}$ & $y^{*++}$ & $\mathbf{y}^{0}$ & $\mathbf{y}^{\mathbf{0 + +}}$ & $\#$ \\
\hline 1 & 58.0287 & 29.5180 & & & & & G & & & & & & & 14 \\
\hline 2 & 157.0972 & 79.0522 & & & & & $\mathbf{V}$ & 1666.7564 & 833.8818 & 1649.7298 & 825.3685 & 1648.7458 & 824.8765 & 13 \\
\hline 3 & 271.1401 & 136.0737 & 254.1135 & 127.5604 & & & $\mathbf{N}$ & $|1567.6879|$ & $\mid 784.3476$ & 1550.6614 & 775.8343 & 1549.6774 & $|775.3423|$ & 12 \\
\hline 4 & 384.2241 & 192.6157 & 367.1976 & 184.1024 & & & I & $|1453.6450|$ & 727.3261 & 1436.6185 & 718.8129 & 1435.6344 & $|718.3209|$ & 11 \\
\hline 5 & 547.2545 & 274.1309 & 530.2279 & 265.6176 & & & M & $|1340.5609|$ & 670.7841 & 1323.5344 & 662.2708 & 1322.5504 & 661.7788 & 10 \\
\hline 6 & 618.2916 & 309.6494 & 601.2650 & 301.1362 & & & $\mathbf{A}$ & $\mid 1177.5306$ & $\mid 589.2690$ & 1160.5041 & 580.7557 & 1159.5201 & 580.2637 & 9 \\
\hline 7 & 765.3600 & 383.1836 & 748.3334 & 374.6704 & & & $\mathbf{F}$ & $\mid 1106.4935$ & 553.7504 & 1089.4670 & 545.2371 & 1088.4830 & 544.7451 & 8 \\
\hline 8 & 916.3539 & 458.6806 & 899.3274 & 450.1673 & & & $\mathrm{C}$ & 959.4251 & 480.2162 & 942.3986 & $\mid 471.7029$ & 941.4145 & $|471.2109|$ & 7 \\
\hline 9 & 1086.4958 & 543.7516 & 1069.4693 & 535.2383 & & & $\mathbf{K}$ & 808.4312 & 404.7192 & 791.4046 & 396.2060 & 790.4206 & 395.7139 & 6 \\
\hline 10 & 1201.5228 & 601.2650 & 1184.4962 & 592.7517 & 1183.5122 & 592.2597 & D & 638.2893 & 319.6483 & 621.2627 & 311.1350 & 620.2787 & 310.6430 & 5 \\
\hline 11 & 1364.5861 & 682.7967 & 1347.5596 & 674.2834 & 1346.5755 & 673.7914 & $\mathbf{Y}$ & 523.2623 & 262.1348 & 506.2358 & 253.6215 & & & 4 \\
\hline 12 & 1478.6290 & 739.8182 & 1461.6025 & $\mid 731.3049$ & 1460.6185 & 730.8129 & $\mathbf{N}$ & 360.1990 & 180.6031 & 343.1724 & 172.0899 & & & 3 \\
\hline 13 & 1549.6661 & 775.3367 & 1532.6396 & 766.8234 & 1531.6556 & 766.3314 & A & 246.1561 & 123.5817 & 229.1295 & 115.0684 & & & 2 \\
\hline 14 & & & & & & & $\mathbf{R}$ & 175.1190 & 88.0631 & 158.0924 & 79.5498 & & & 1 \\
\hline
\end{tabular}




\section{AT2G33090.1}

SK $_{79}$ TGEVTREK, expmz 1175.657808

Interpreted with: K2, Trimethyl (K)
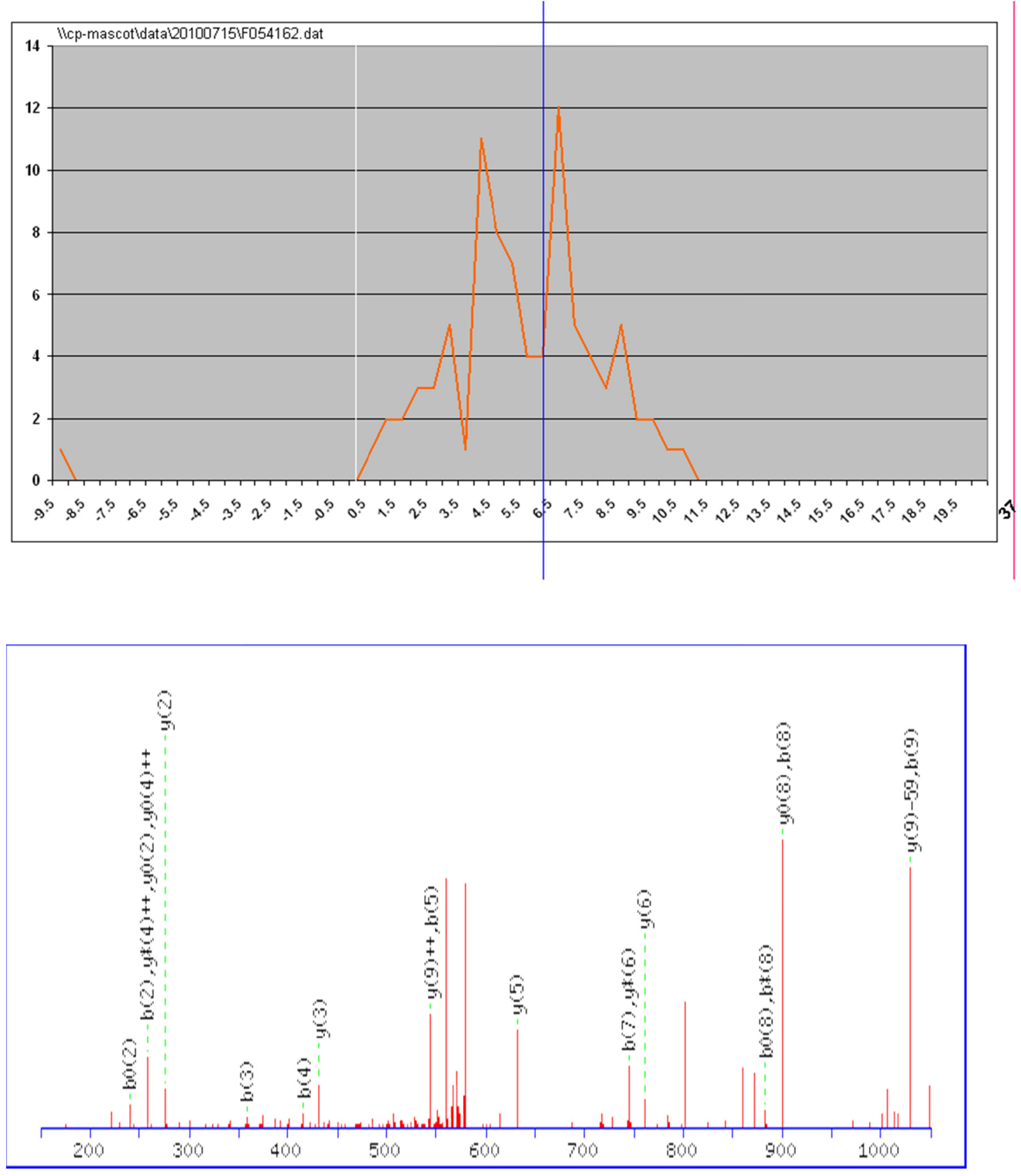


\begin{tabular}{|c|c|c|c|c|c|c|c|c|c|c|c|c|c|c|}
\hline$\#$ & b & $\mathbf{b}^{++}$ & $\mathbf{b}^{*}$ & $\mathbf{b}^{*++}$ & $\mathbf{b}^{0}$ & $\mathbf{b}^{0++}$ & Seq. & $\mathbf{y}$ & $\mathbf{y}^{++}$ & $y^{*}$ & $y^{*++}$ & $\mathbf{y}^{0}$ & $\mathbf{y}^{\mathbf{0}^{++}}$ & \# \\
\hline 1 & 88.0393 & 44.5233 & & & 70.0287 & 35.5180 & $\mathrm{~S}$ & & & & & & & 10 \\
\hline 2 & 258.1812 & 129.5942 & 241.1547 & $\mid 121.0810$ & 240.1707 & 120.5890 & K & 1089.6263 & 545.3168 & 1072.5997 & 536.8035 & 1071.6157 & $\mid 536.3115$ & 9 \\
\hline 3 & 359.2289 & 180.1181 & 342.2023 & 171.6048 & 341.2183 & 171.1128 & $\mathbf{T}$ & 919.4843 & 460.2458 & 902.4578 & 451.7325 & 901.4738 & 451.2405 & 8 \\
\hline 4 & 416.2504 & 208.6288 & 399.2238 & 200.1155 & 398.2398 & 199.6235 & G & 818.4367 & 409.7220 & 801.4101 & 401.2087 & 800.4261 & 400.7167 & 7 \\
\hline 5 & 545.2930 & 273.1501 & 528.2664 & 264.6368 & 527.2824 & 264.1448 & $\mathbf{E}$ & 761.4152 & 381.2112 & 744.3886 & 372.6980 & 743.4046 & 372.2060 & 6 \\
\hline 6 & 644.3614 & 322.6843 & 627.3348 & 314.1710 & 626.3508 & 313.6790 & V & 632.3726 & 316.6899 & 615.3461 & 308.1767 & 614.3620 & 307.6847 & 5 \\
\hline 7 & 745.4090 & 373.2082 & 728.3825 & $\mid 364.6949$ & 727.3985 & 364.2029 & $T$ & 533.3042 & 267.1557 & 516.2776 & 258.6425 & 515.2936 & 258.1504 & 4 \\
\hline 8 & 901.5102 & 451.2587 & 884.4836 & 442.7454 & 883.4996 & 442.2534 & $\mathbf{R}$ & 432.2565 & 216.6319 & 415.2300 & 208.1186 & 414.2459 & 207.6266 & 3 \\
\hline 9 & 1030.5528 & 515.7800 & 1013.5262 & $|507.2667|$ & $\mid 1012.5422$ & 506.7747 & $\mathbf{E}$ & 276.1554 & 138.5813 & 259.1288 & 130.0681 & 258.1448 & 129.5761 & 2 \\
\hline 10 & & & & & & & $\mathbf{K}$ & 147.1128 & \begin{tabular}{|l|}
74.0600 \\
\end{tabular} & 130.0863 & \begin{tabular}{|l|}
65.5468 \\
\end{tabular} & & & 1 \\
\hline
\end{tabular}

